\title{
Grid Based Sensor Node Deployment Approach for Localization in WSN
}

\author{
Priyanka Rajput ${ }^{1}$ and Madhukar Dubey ${ }^{2}$ \\ M.tech. Dept. of Computer Science \& Engineering ${ }^{1}$ \\ M.tech. Dept. of Computer Science \& Engineering ${ }^{2}$ \\ priyankarjpt32@gmail.com ${ }^{1}$ \\ madhukardubey@hotmail.com ${ }^{2}$
}

\begin{abstract}
Localization is widely exploited in Wireless Sensor Networks (WSNs) to apprehend the existing locality of the sensor nodes. Awareness of location is a vital and critical problem and challenge in WSN. Information of locality among the participating nodes is a vital necessity in designing of solutions for several problem related to WSN. WSN are being utilized in atmosphere applications to perform the many task e.g. atmosphere monitoring, target tracking, defenses and various more. In numerous jobs, localization node is inherently machine parameters. Localization Node is necessary to report the dispatcher of activities, assist querying set of sensors, routing and to solution queries on the network coverage. Thus, one of the foremost challenges in WSN is node localization. In this paper we study about sensor network and localization technique we proposed a grid based approach to find best location of sensor nodes. Our work has been implemented on NS2.35 and our results show that our work is better than the existing work for the efficient location of nodes in the network.
\end{abstract}

Keywords: WSN; Localization; Localization parameter; localization algorithm.

\section{Introduction}

In WSNs, sensor nodes (Nodal) are placed in actual atmosphere and decide a few physical behaviors. WSNs have several challenges of research. Sensors are the tiny tool, lowest costing, and having low processing abilities. WSNs application attracted first-rate interest of researchers in latest years [1]. WSNs are dissimilar from ad hoc and mobility networks in numerous ways. WSNs have unique applications; so, the protocols planned for ad hoc networks don't suit WSNs [2]. Dissimilar programs of WSNs are the subsequent: tracking environmental components and bodily phenomena like light, habitat patient healthcare observe. WSNs have various study problems that affect performance and design of overall network e.g. hardware and operating system [3], medium access schemes [4], deployment [5], time synchronization [6], localization, middleware, wirelessly sensors and actors networks [7], transport layer, network layer, quality of service, and network security [8]. WSNs applications have opened difficult and innovative studies areas in telecommunication global specifically in previous few years. 


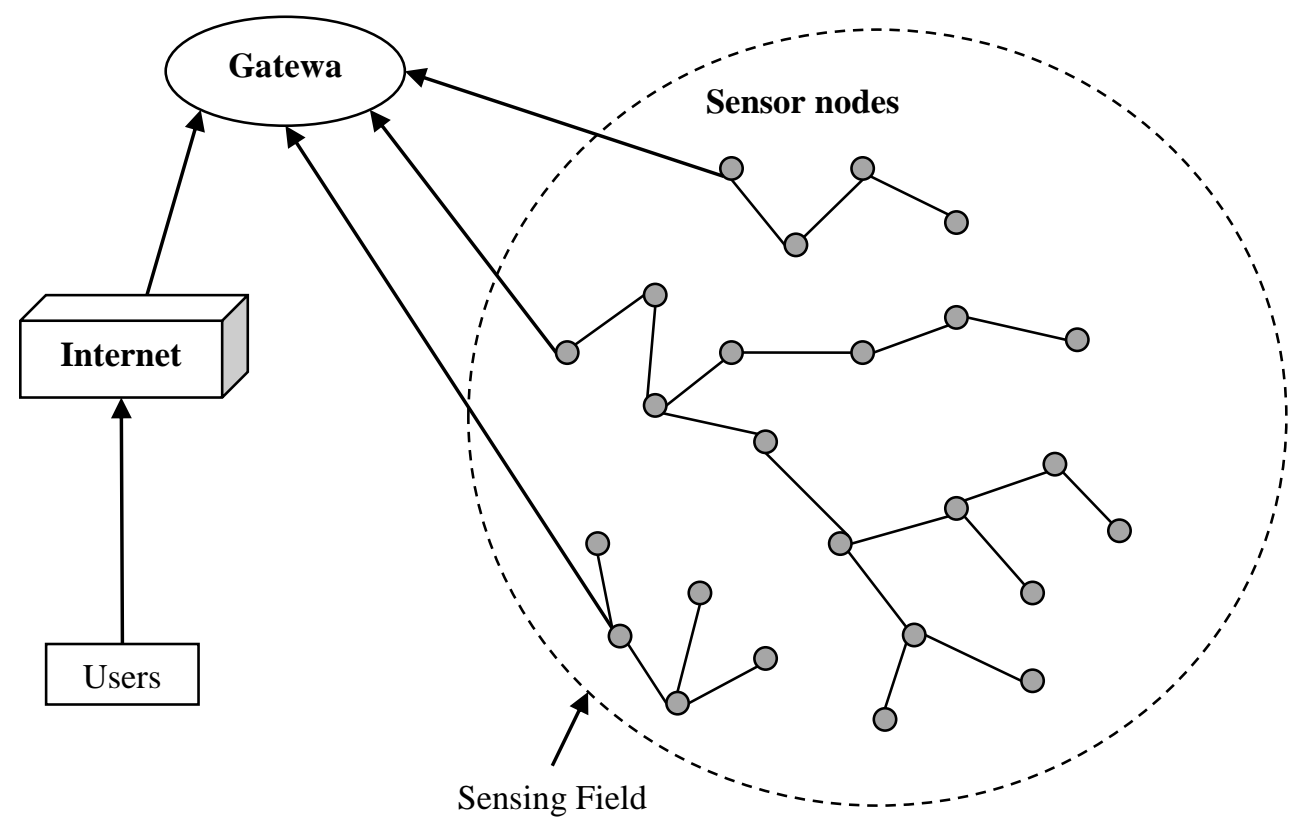

Figure 1. WSN Architecture

Localization nodal are very significant to detect and decide locality of sensor node with the aid of a specialized set of rules. Localization is the method of detecting the nodal position [9] as info and statistics are otiose if the nodal have no concept in their geographical positions. GPS (global positioning system) is the good approach for localization of nodal, but it becomes very expensive if a biggest range of nodal occurs in a specified network. Many algorithms had been defining to solve the issues of localization; however, most of the existing algorithms are application unique and maximum of the solutions aren't proper for huge form of WSNs $[10,11]$. Ultra extensive band plans are suitable for indoor surroundings whilst acoustic transmission-primarily based system calls for greater hardware. Both are accurate techniques but expensive in terms of energy consumption and processing. Various algorithms have been presented to minimalize this communiqué price. If one node estimates its incorrect position, then this error propagates to overall network and next nodal; as a outcome, incorrect info of anchor nodal location is propagated. To detect the locality of nodal is mostly depend on distance amid unlocalized node (with unknown location) and anchor node (with known location) [12].

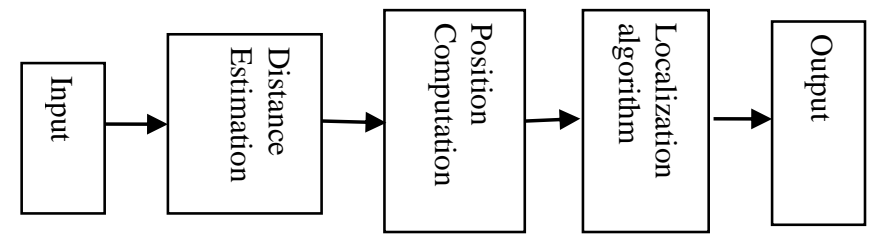

Figure 2. Localization Procedure

\section{Parameter of Localization}

For the dissimilar ways of approximating locality info, we've to name parameters to differentiate the similarities and dissimilarity amongst unique approaches. We present the utmost typical parameters to classify distinctive strategies. 


\subsection{Accuracy}

Accuracy is very vital in the localization of WSN. Higher accuracy is generally essential in military installations, simply as sensor $\mathrm{n} / \mathrm{w}$ deployed for intrusion detection. However, for commercial $\mathrm{n} / \mathrm{w}$ that may utilize localization to convey advertisements from neighboring shops, the required accuracy may not be lower.

\subsection{Cost}

Cost is a totally tough affair within the localization of WSN. There are only certain algorithms which offer low cost however the ones algorithms don't give the high charge of accuracy

\subsection{Power}

Power is vital for computation purpose. Power play a critical role in WSN as each sensor tool has restrained electricity. Power provided via battery.

\subsection{Static Nodes}

In atmosphere, all static sensor nodal are analogous. This means that, each the nodal have same sensing ability, estimating capability, and the ability to communicate. We as well think that, the preliminary battery powers of the nodal are equal at deployment.

\subsection{Mobility Nodes}

It's supposed that a few wide varieties of GPS enabled mobility nodal are part of the sensor $\mathrm{n} / \mathrm{w}$. These nodal are homogeneous in nature. But, are presumed to have more battery power in comparison to the static nodal and don't drain out totally all thru the localization procedure. The communiqué variety of mobility sensor nodal are assumed no longer to exchange drastically for the duration of the complete localization algorithm runtime and also not to modification meaningfully within the reception of four beacon messages thru a specific static node [13].

\section{Localization Algorithm}

The localization algorithm can be broadly classified into two classes:

\subsection{Range Based}

In range based totally mechanisms, the locality of a sensor node can be decided with the aid of the angle or distance metrics. These metrics are TDoA (Time Difference of Arrival), RSSI (Received Signal Strength Indicator), AoA (Angle of Arrival), ToA (Time of Arrival). Range based totally method are distinctly correct however, they're geared up with incredibly high-priced hardware and necessitates quite a few computation. It rises the costly of the $\mathrm{n} / \mathrm{w}$ and is inefficient in phrases of computations. The several varieties based whole strategies are Radio Interferometry Measurement (RIM), Multidimensional Scaling (MDS), 3D - Landscape, DV-distance, DV-hop, Euclidean distance etc.

\subsection{Range Free}

In this method, the sensor node locality is identified on the basis info transmitted thru neighboring node; rely on hop or on triangulation foundation. The various range free approach are APIT, chord election technique, 3-D multil-ateration method, centroid pattern etc. The range free technique has a fault in accuracy up to $10 \%$ of the communiqué range of separate node. But, these approach are much cheaper than the range based approach [14]. 


\subsection{Anchor free and Anchor based}

Nodal of anchor are the one's nodal which apprehend their coordinates via GPS or manual region. Thru exploit of anchor nodal we are able to get worldwide coordinates but issue of exploiting anchor nodal is that GPS receivers are highest expensive and mightn't be exploited inner for localization due GPS make utilize of LoS communiqué. Other to GPS is preprogrammed nodal with their locality that is impractical or even not possible.

For inner localization, we will utilize anchor free nodal and might actually get relative coordinates or we can utilize few anchor based nodal that understand their coordinates thru manual locality. Anchor Free or Anchor Based approach are given in Table I.

\section{Table 1. Classification of Proposals for Localization in WSN [15]}

\begin{tabular}{|l|l|l|}
\hline PROPOSALS & $\begin{array}{l}\text { Anchor Based/ } \\
\text { Anchor Free }\end{array}$ & $\begin{array}{l}\text { Range Based/Range } \\
\text { Free }\end{array}$ \\
\hline Miao Jin, et al., & Anchor Free & Range Free \\
\hline KoenLangendoen, et al., & Anchor Based & Both \\
\hline E. S. Navarro, et al., & Anchor Based & Range Free \\
\hline Yi Shang, et al., & Anchor Based & Range Free \\
\hline Yi Shang, et al., & Anchor Free & Range Free \\
\hline Anushiya A Kannan, et al. & Anchor Based & Range Free \\
\hline C. Alippi, et al., & Anchor Based & Range Based \\
\hline T. He, et al., & Anchor Based & Range Free \\
\hline A. Savvides, et al., & Anchor Based & Range Based \\
\hline S. Simic, et al. , J. Bachrach, et al., & Anchor Based & Range Free \\
\hline J. & Anchor Free & Range Free \\
\hline
\end{tabular}

\section{Literature Survey}

Chun-Yi Wei et al. (2017) in this paper, In this work, we demonstrate the significance of threshold design for accurate and robust target localization in WSN, particularly when the various sensors is finite. With this goal, we derive a higher bound on the probability of misclassification as a characteristic of RSS thresholds with the aid of the usage of the union inequality. The RSS thresholds that algorithmically diminish the derived misclassification fault bound are then numerically received over a reflect- based holomorphic sensor diffuse building. Simulations over fading wirelessly links display that the scheme based totally on newly observed optimized RSS thresholds appreciably outperforms the preceding scheme the utilize of the thresholds which might be intuitively selected, especially in the presence of Byzantine attacks that severely impact information security [16].

Kan Zhenget. al (2016) in this paper, they define an Tracking (eLOT) and energyefficient Localization system, utilizing lowest-prise and portable $\mathrm{h} / \mathrm{w}$ to enable highly accurate tracking of targets. Several fingerprint-depend method for tracking and localization are implemented in eLOT. In sequence to achieve highest power efficiency, a n/w-level scheme coordinating interference and collision is define. On another hand, depend on the locality info, mobility devices in eLOT can rapidly associate with the exact channel in a provide region, while saving energy through evading needless communication. Finally, a platform depend on TI CC2530 and the Linux operating system is built to show the usefulness of our define scheme in terms of localization accurateness and energy efficiency [17].

Shi Zhang et. al (2016) in this paper,an Extended Kalman Filter Multidimensional Scaling (EKF-MDS) localization algorithm is proposed. Thru discovering the virtual node and building the shortest paths amid nodal, the Euclidean distances amid nodal 
are achieved. The Extended Kalman Filter (EKF) is applied to refine the locality coordinates that are achieved thru the MDS-MAP algorithm. Simulation results demonstrate that the define algorithm is exceedingly accurate and efficient comparing to state-of-the-art methods in anisotropic networks with holes[18].

XiaofengXu, YongjiRen (2016) In this paper, they define a newest info game based secure localization method for WSN. The secure localization difficulty in opposed scenario has been transformed into an data game issue. Simulation outcomes demonstration that the define approach is effective to secure localization [19].

SlavisaTomic et al. (2016) In this paper, they define a three-step procedure in sequence to enhance the estimation accuracy of our algorithms. The simulation outcome confirmed the efficiency of the most up-to-date algorithms in a selection of settings. For the case of non-cooperative localization, the outcomes presented that the define techniques significantly outperform the present one, even for the case where the define estimators have no knowledge about PT. For the case of cooperative localization, they have examined the influence of $\mathrm{N}, \mathrm{M}$ and $\mathrm{R}$ on the estimation accuracy. For all considered scenarios, the novel estimators exhibited brilliant performance, and robustness to not knowing PT [20].

N.M. Ngabas et al. (2016) In this paper, they define a newest algorithm by implementing a selective forwarding during localization phase in original range-free localization algorithm named DV-hop. In the define scheme, both energy and accuracy level are taken into consideration before the sensor node forwards the packet in the $\mathrm{n} / \mathrm{w}$. They adopted Markov Decision Process (MDP) idea for a sensor to perform elective forwarding. The results show the selective forwarding improves the average energy level, diminish the duplicated packet and didn't diminish the accuracy level [21]

Vaishali R. Kulkarni et al. (2016) In this paper, Many swarm intelligence algorithms have been define for accurate localization. The unused huge potential of the artificial bee colony ( $\mathrm{ABC}$ ) algorithm has inspired the investigation presented in this paper. The $\mathrm{ABC}$ algorithm has been investigated as a tool for anchor-assisted sensor localization in WSNs. Result of Matlab simulation of ABC-based multistage localization has been presented. Additional, the outcomes are equated with those of the localization approach rely on the PSO algorithm. The performances of PSO and $\mathrm{ABC}$ algorithms has been presented in terms of the no. of nodal localized, localization the computation time and accuracy. The outcomes show that the ABC algorithm delivers higher accuracy of localization than the PSO algorithm does; but, it takes longer to converge. This outcomes in a trade-off amid accuracy and speed of localization in WSNs [22].

\section{Proposed Work}

In our proposed work first we introduce sensor area as grid and find in one grid how many nodal are present than we Deploy one mobility node in every grid. After the deployment mobility node behaves as grid member .Each mobility node connected to each other and knows their location. With the aid of mobile coordinate all nodal get their coordinate. 
Proposed Model:

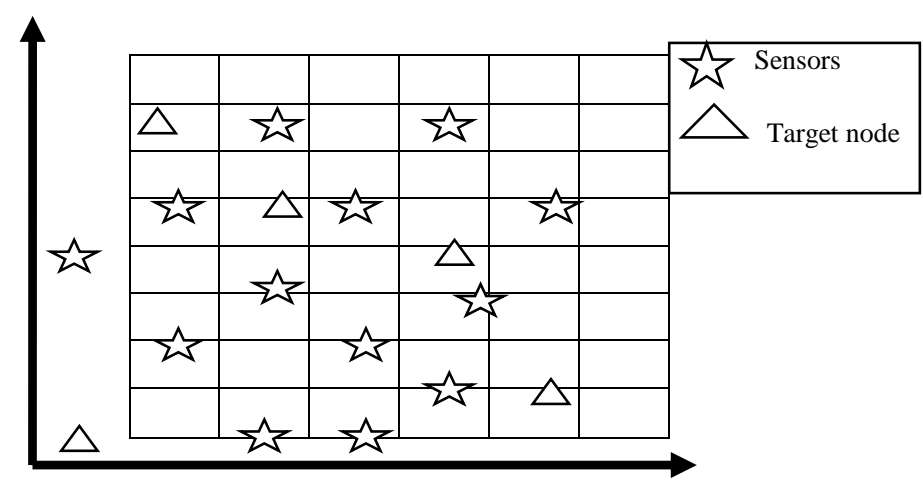

Figure 3. The Diagram of Network

Sensor nodes randomly extend in the network. Each node is location-aware and static and; destinations are also in the static state. For the ease of study, the monitor region is defined as the square of area of $n \times n$, it's separated into $N(N=n \times n)$ grids. $M(M<<N)$ sensor nodes with position data are randomly deploy within few grids.

Considering the effectiveness of network coverage (the Monitored region is covered thru a least number of sensors), we suppose that there's at most of one node for every grid. $K$ $(K<M<<N)$ target are scattered in dissimilar grids, and there is no more than one target in every grid. Moreover, the real target's position is supposed as the similar grid center. The layout of the network is illustrated as Fig. 1. 


\section{Flow diagram:}

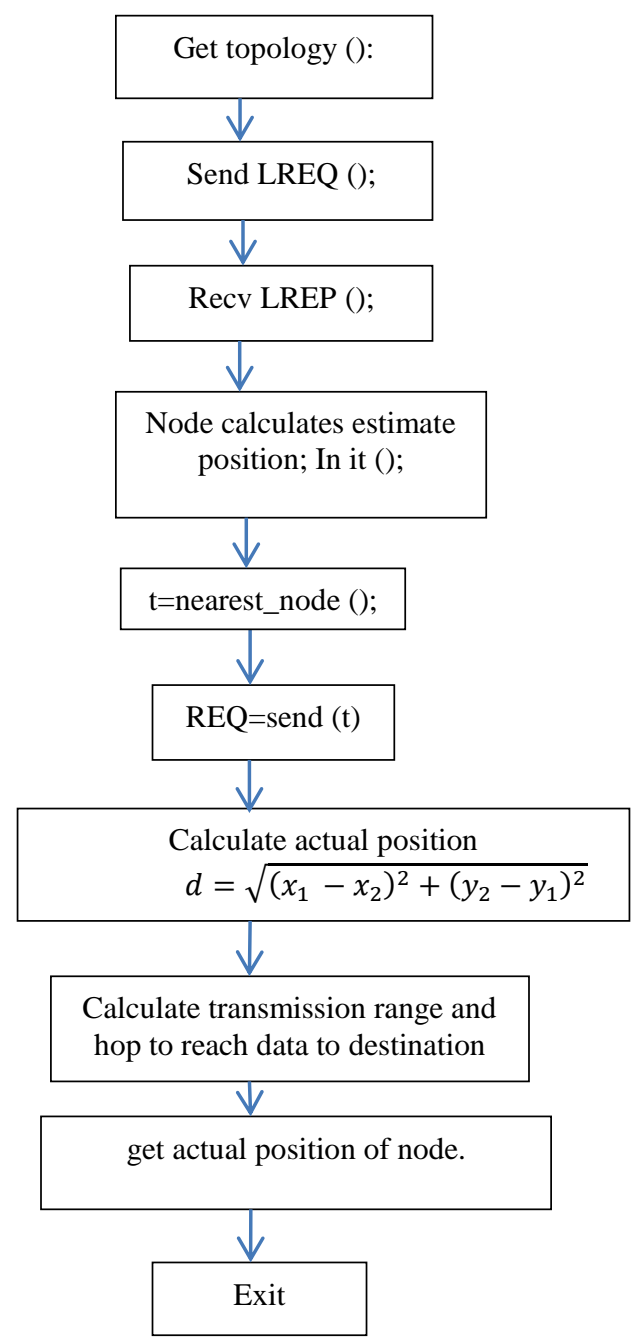

Figure 4. Flow Diagram

\section{Performance Evaluation}

We simulated the localization approach of energy efficient on NS (version 2) broadly recognized, a scalable discrete event driven simulation tool. Building highest performance WSN systems necessitates an understanding of the activities of sensor network and what create them slowly or rapid. In addition to the performance analysis, we have also evaluated the proposed technique in measuring, evaluating, and understanding system performance.

\section{Simulation \& Results}

The simulation is carried out in NS2. The number of nodes is 50, XY dimension is $2000 \mathrm{X} 2000$. 
Table 2. Simulation Parameters

\begin{tabular}{|l|l|}
\hline Topology Size & $2000 \mathrm{~m} \mathrm{X} 2000 \mathrm{~m}$ \\
\hline Number of node & 50 \\
\hline $\begin{array}{l}\text { Number of } \\
\text { unknown node }\end{array}$ & 2 \\
\hline Beacon node & 3 \\
\hline MAC layer & IEEE 802.11 \\
\hline Protocol & AODV \\
\hline X & 2000 \\
\hline Y & 2000 \\
\hline Simulation Time & 50 sec \\
\hline Traffic Source & $\begin{array}{l}\text { Constant bit } \\
\text { rate }(\mathrm{CBR})\end{array}$ \\
\hline Packet Size & 512 bytes \\
\hline Transmit Power & $360 \mathrm{~mW}$ \\
\hline Range & $500 \mathrm{~m}$ \\
\hline Node Placement & Random waypoint \\
\hline
\end{tabular}

\subsection{Packet Delivery Ratio (PDR)}

The ratio of packets delivered from source to endpoint. The Fig illustrates a PDR graph amid proposed approach and base approach. The proposed approach is better than the base approach to the PDR.

$P D R=\frac{\text { total no of packets received }}{\text { total no of packets send }}$

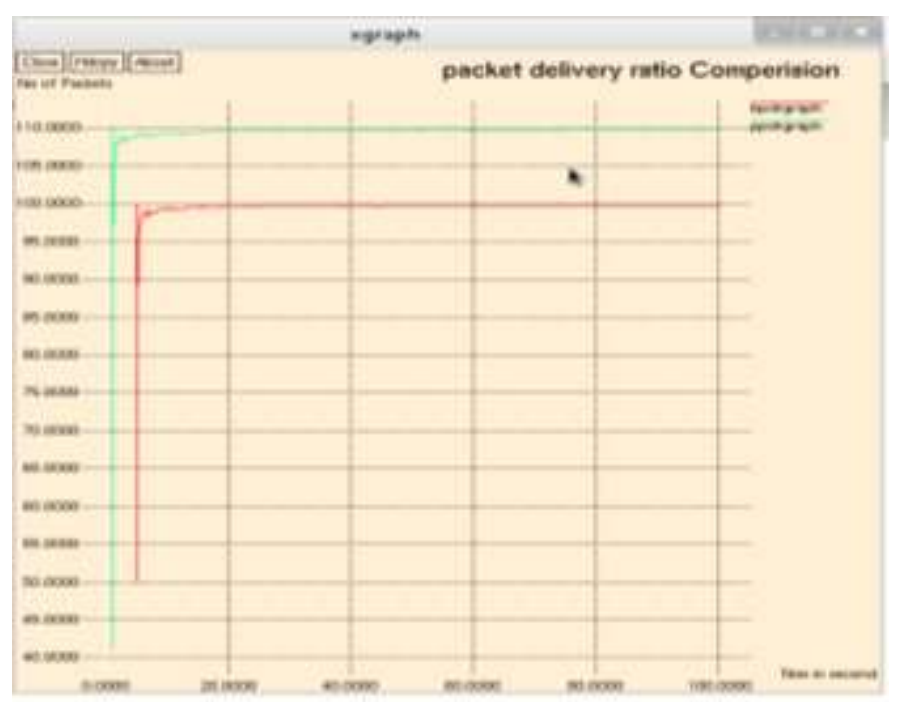

Figure 5. PDR Graph 


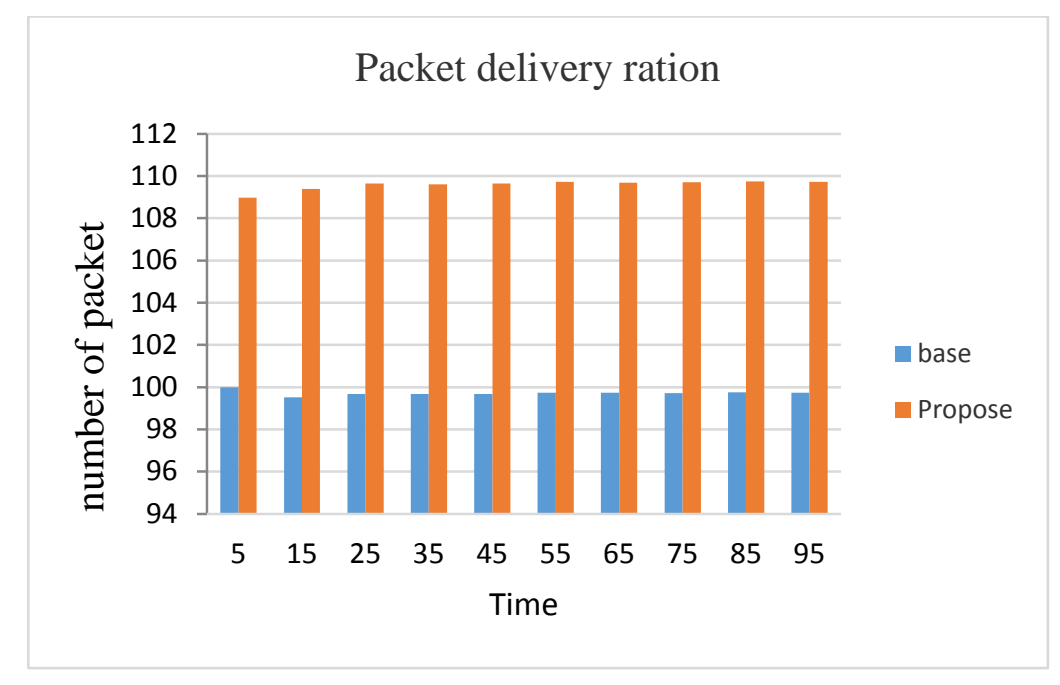

Figure 6. PDR Value of Graph 5

\subsection{Routing Overhead}

The routing overhead is described as flooding data and data of data and in the network sent thru application, which utilizes a bit of available transfer rate of communiqué protocols. The Fig. 4 represents a routing overhead graph amid proposed approach and base approach. Since the overhead should be least but as the routing improvements in the proposed work, the overhead also improves.

Routing overhead $=\frac{\text { number of flooding data }}{\text { time }}$

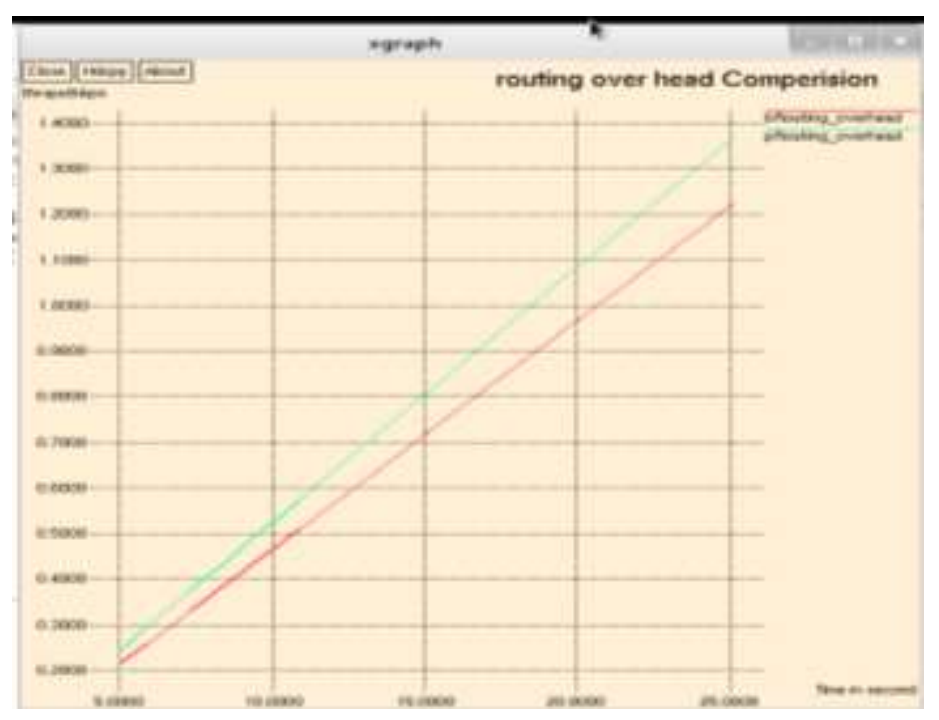

Figure 7. Routing Overhead Graph 


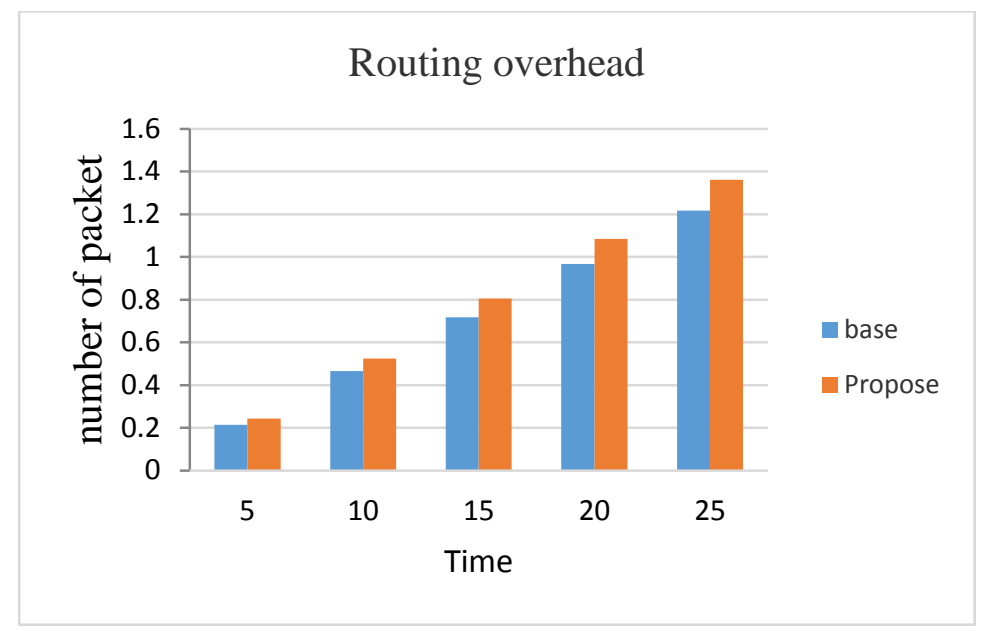

Figure 8. Routing Overhead Value of Graph 7

\subsection{Throughput}

Throughput of the network is calculated thru the whole no. of packets sent over a particular period of time. In this graph, it is shown that the throughput increase as compared to existing works.

Transmission Time $=\frac{\text { File Size }}{\begin{array}{l}\text { Band width }(\mathrm{sec}) \\ \text { File Size }\end{array}}$

Throughput $=\frac{\text { File Size }}{\text { Transmission Time (bps) }}$

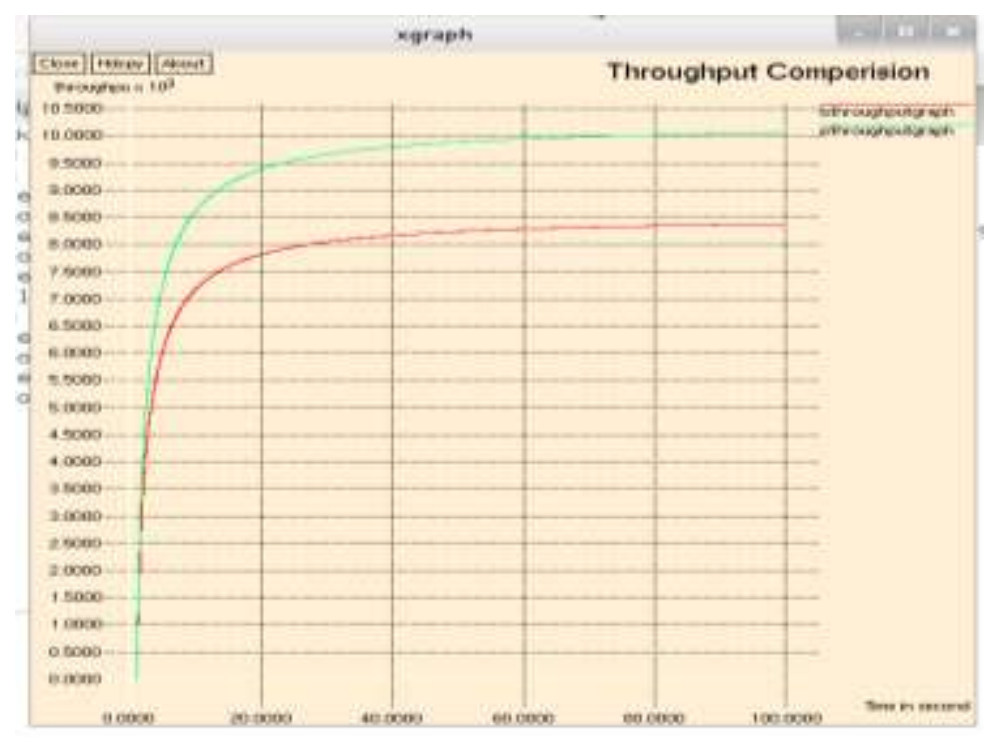

Figure 9. Throughtput Graph 


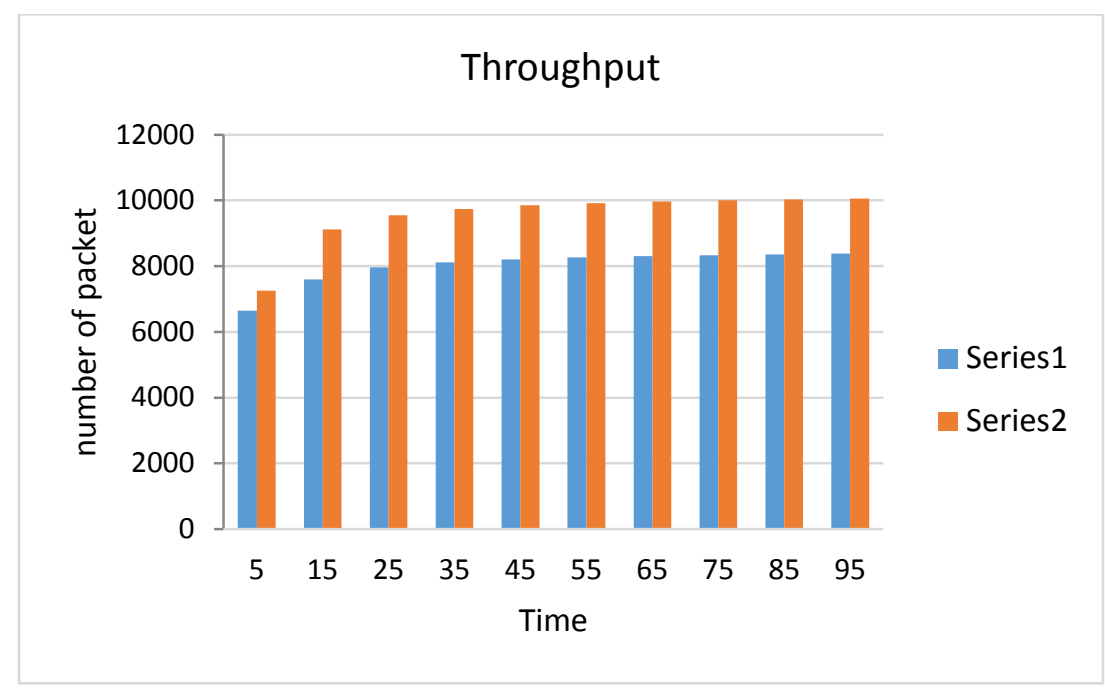

Figure 10. Throughtput Value of Graph 9

Table 3.Comparison Table

\begin{tabular}{|l|l|l|l|}
\hline PARAMETERS & RANGE & power & Accuracy \\
\hline RANGE-BASED & $\begin{array}{l}\text { long range } \\
\text { calculation using } \\
\text { each node }\end{array}$ & HIGH POWER & HIGH \\
\hline RANGE-FREE & $\begin{array}{l}\text { long range using } \\
\text { db hope and } \\
\text { distance }\end{array}$ & MODERATE & HIGH \\
\hline WITH ANCHOR - & $\begin{array}{l}\text { range and angle } \\
\text { NODE techniques } \\
\text { and TOA LOW }\end{array}$ & $\begin{array}{l}\text { HIGH USING } \\
\text { GPS AND } \\
\text { ANCHOR-NODE }\end{array}$ \\
\hline WITHOUT \\
ANCHOR -NODE & $\begin{array}{l}\text { range detect using } \\
\text { GPS LOW }\end{array}$ & $\begin{array}{l}\text { AVERAGE } \\
\text { USING ONLY } \\
\text { GPS }\end{array}$ \\
\hline GRID BASED & $\begin{array}{l}\text { high } \\
\text { predicting rangr } \\
\text { neighbour grids }\end{array}$ & LOW & $\begin{array}{l}\text { HIGH,EXACT } \\
\text { AND EFFECTIVE }\end{array}$ \\
\hline
\end{tabular}

\section{Conclusion}

Wireless ad-hoc sensor networks have as of late developed as a premier research subject. They have extraordinary long term capacity to modify our lives, economic potential, and pose various new system-building issues. Sensor networks also possessing a certain innovative optimization and conceptual problem. For instance, namely position, consumption, and tracing, are essential issues, various applications relying on them requiring information. Localization is used for tracking the actual position of nodes. The results of proposed approach are better in terms of PDR and routing overhead and throughput.

In our future work, we can deploy multiple mobile nodes in the grid area to enhance the scalability of the network. We utilized distance calculation approach for the evaluation of transmission range for data transmission. This method is very efficient but we can use more enhanced method to decrease the computational complexity. Then apply security to 
the data which improved the performance of the sensor network by using secure and verified localization technique.

\section{References}

[1] I. F. Akyildiz, W. Su, Y. Sankarasubramaniam and E. Cayirci, "Wireless sensor networks: a survey", PII: S1389-1286(01)00302 -4, Published by Elsevier, (2002).

[2] J. Yick, B. Mukherjee and D. Ghosal, "Wireless sensor network survey", Elsevier, vol. 52, no. 12, (2008), pp. 2292-2330.

[3] S. Gowrishankar, T. G. Basavaraju, D. H. Manjaiah and S. K. Sarkar, "Issues in wireless sensor networks", Proceedings of the World Congress on Engineering, (2008); London, U.K

[4] C. Vehbi, C. Gungor, B. Lu and G. P. Hancke, "Opportunities and challenges of wireless sensor network in smard grid", vol. 57, no. 10, (2010).

[5] D. Ganesan, A. Cerpa, W. Ye, Y. Yu, J. Zhao and D. Estrin, "Networking issues in wireless sensor networks", Published by Elsevier, vol. 64, no. 7, (2004), pp. 799-814.

[6] Y.-C. Wu., Q. Chaudhari and E. Serpedin, "Clock synchronization for wireless sensor networks", IEEE, vol. 28, no. 1, (2011).

[7] I. F. Akyildiz and I. H. Kasimoglu., "Wireless sensor and actor networks", Research Challenges Ad Hoc Networks", Elsevier, (2004).

[8] J. A. Stankovic, "Research challenges for wireless sensor networks", ACM, vol. 1, no. 2, (2004), pp. 912.

[9] G. Mao, B. Fidan and B. D. O. Anderson, "Wireless sensor network localization techniques", Elsevier, vol. 51, no. 10, (2007), pp. 2529-2553.

[10] A. Savvides, C.-C. Han and M. B. Strivastava, “.Dynamic fine-grained localization in ad-hoc networks of sensor", ACM, (2001), pp. 166-179.

[11] A. Savvides, H. Park and M. B. Srivastava, "The n-Hop Multilateration Primitive for Node Localization Problems", Spinger, vol. 8, no. 4, (2003), pp. 443-451.

[12] N. Ali Alrajeh, M. Bashir and B. Shams, "Localization Techniques in Wireless Sensor Networks", sage journal, vol. 9 no. 6, (2013).

[13] S. Aggarwal and V. Gupta, "Localization in Wireless Sensor Networks", IJARECE, vol. 5, no. 5, (2016).

[14] V. Yadav, M. K. Mishra, A. K. Sngh and M. M. Gore, "Localization Scheme for Three Dimensional Wireless Sensor Networks Using GPS Enabled Mobile Sensor Nodes”, IJNGN, vol. 1, no. 1, (2009).

[15] U. Nazir, M. A. Arshad, N. Shahid and S. H. Raza, "Classification of Localization Algorithms for Wireless Sensor Network: A Survey", IEEE, (2012).

[16] C.-Y. Wei, P.-N. Chen, Y.-S. S. Han and P. K. Varshney, "Local Threshold Design for Target Localization using Error Correcting Codes in Wireless Sensor Networks in the Presence of Byzantine Attacks", 1556-6013 (c) IEEE, (2016).

[17] K. Zheng, H.-J.Wang, H. Li, W. Xiang, J. Qiao and X. (Sherman) Shen, "Energy-Efficient Localization and Tracking of Mobile Devices in Wireless Sensor Networks", 0018-9545 (c) IEEE, (2016).

[18] S. Zhang, B.-H. Zhang, M. Joo Er and Z.-X. Guan, "A Novel Node Localization Algorithm for Anisotropic Wireless Sensor Networks with Holes Based on MDS-MAP and EKF”, 978-1-5090-2597IEEE, (2016).

[19] X.-F. Xu and Y.-J. Ren, "Information Game Model for Secure Localization in Wireless Sensor Networks", 978-1-5090-0729-5/16/\$31.00 OIEEE, (2016).

[20] S. Tomic, M. Beko and R. Dinis, "3-D Target Localization in Wireless Sensor Network Using RSS and AoA Measurements”, 0018-9545 (c) IEEE, (2016).

[21] N. M. Ngabas, J. Abdullah, R. Alias, A. Jamil and L. Audah, "Selective Forwarding in DV-Hop Localization of Wireless Sensor Networks", 978-1-5090-6100-6116/\$31.00 @ IEEE, (2016).

[22] V. R. Kulkarni, V. Desai and R. V. Kulkarni, "Multistage Localization in Wireless SensorNetworks using Artificial Bee Colony Algorithm”, 978-1-5090-4240-1/16/\$31.00 @ IEEE, (2016). 